\title{
Criminologie
}

\section{Victimes d'actes criminels : une intervention professionnelle}

\section{Frema Engel}

Volume 23, numéro 2, 1990

Après le crime : survivre

URI : https://id.erudit.org/iderudit/017292ar

DOI : https://doi.org/10.7202/017292ar

Aller au sommaire du numéro

Éditeur(s)

Les Presses de l'Université de Montréal

ISSN

0316-0041 (imprimé)

1492-1367 (numérique)

Découvrir la revue

Citer cet article

Engel, F. (1990). Victimes d'actes criminels : une intervention professionnelle. Criminologie, 23(2), 5-22. https://doi.org/10.7202/017292ar

\section{Résumé de l'article}

Victims of crime suffer tremendous psychological damage as a result of the trauma that they experience. The effects can be totally debilitating and can destroy their lives. The author discusses the reactions, symptoms of acute stress and stages of recovery of crime victims. She also discusses post-traumatic stress syndrome (PTSD), and the kind of help victims need to recover from their injuries. Finally, she suggests how mental health professionals in particular and society in general could better respond to crime victims.
Ce document est protégé par la loi sur le droit d'auteur. L'utilisation des services d'Érudit (y compris la reproduction) est assujettie à sa politique d'utilisation que vous pouvez consulter en ligne.

https://apropos.erudit.org/fr/usagers/politique-dutilisation/ 


\begin{abstract}
Victims of crime suffer tremendous psychological damage as a result of the trauma that they experience. The effects can be totally debilitating and can destroy their lives. The author discusses the reactions, symptoms of acute stress and stages of recovery of crime victims. She also discusses post-traumatic stress syndrome (PTSD), and the kind of help victims need to recover from their injuries. Finally, she suggests how mental health professionals in particular and society in general could better respond to crime victims.
\end{abstract}

\title{
INTRODUCTION
}

Dans toutes les villes nord-américaines, la fréquence des crimes violents devient alarmante. En 1988, dans la province de Québec, on a recensé 44129 crimes contre la personne. En 1989, ce nombre est passé à $46717^{\prime}$. Dans la seule ville de Montréal, en 1989, 23265 crimes contre la personne ont été rapportés, ce qui représente une augmentation par rapport à 1988, où l'on dénombrait 22263 crimes de cette nature. Ceux-ci regroupent les agressions, les.attaques à main armée, les agressions sexuelles, les tentatives de meurtre et les homicides ${ }^{2}$.

Malgré le nombre de gens touchés par l'acte criminel, et l'étalage médiatique d'images et d'histoires horribles, nous nous détournons des victimes qui ont survécu à un acte criminel. Elles composent un groupe de notre société qui est privé de ses droits. Leurs besoins sont ignorés, nous leur refusons souvent de l'aide et, la plupart du temps, nous les laissons se débrouiller comme elles le peuvent après l'agression.

Dans bien des cas, le crime subi par les victimes constitue l'événement le plus perturbant et le plus dommageable de leur vie. Elles souffrent d'une terrible détresse émotive, endurent des pertes financières et, pour plusieurs, le crime détruit complètement leur existence.

Dans cet article, l'auteure explore l'impact du crime sur les victimes. Selon elle, la plupart des victimes d'actes criminels sont atteintes par le syn-

* L'auteure est une travailleuse sociale de pratique privée, spécialisée dans le traitement des victimes d'actes criminels. Engel \& Associés, 4060, rue Ste-Catherine Ouest, Bureau 530, Montréal, Québec, H3Z 2 Z3. 
drome du choc post-traumatique, un ensemble de symptômes psychologiques qui surviennent à la suite d'un traumatisme soudain et aigu. L'auteure est d'avis que notre société minimise la souffrance des victimes d'actes criminels, que le syndrome du choc post-traumatique est peu et mal connu et que, de ce fait, les médecins et les professionnels de la santé posent souvent le mauvais diagnostic et ne fournissent pas aux victimes le traitement qui leur convient. En outre, trop souvent les victimes d'actes criminels n'ont pas accès à l'aide et aux services indispensables au recouvrement de leur santé.

\section{L'EFFET DÉVASTATEUR DU CRIME DANS LA VIE DES GENS}

Le massacre de l'Université de Montréal, le 6 décembre 1989, où 14 jeunes femmes ont trouvé la mort et où 13 autres personnes ont été grièvement blessées, a profondément bouleversé notre société. Ce crime a atteint la vie de beaucoup de gens: les familles et les ami-e-s des étudiantes assassinées, les blessées ainsi que leur famille et leurs ami-e-s, leurs camarades de l'École polytechnique et la communauté de l'Université de Montréal dans son ensemble, professeur-e-s et employé-e-s inclus.

L'Université a réagi rapidement, mettant à la disposition de la communauté estudiantine, de l'équipe d'employé-e-s et des parents, un service d'aide psychologique étendu pour les aider à se remettre du choc et de la peine qu'ils éprouvaient. Malgré les répercussions importantes de ce massacre, la communauté universitaire a eu, dans un sens, de la chance. Les gens se sont rassemblés. Ils se sont réconfortés les uns les autres. Ils ont reçu un immense soutien. Chaque personne qui le désirait ou en éprouvait le besoin pouvait bénéficier d'une aide professionnelle. Ces interventions sont essentielles pour le rétablissement des victimes d'actes criminels ainsi que pour celui de leur famille.

Cependant, malgré les efforts et les exceilents services qui ont été offerts aux victimes, on constatera à long terme que beaucoup d'entre elles, touchées directement ou indirectement par cette tragédie, mettront du temps à se remettre de leurs blessures psychologiques. Alors que certaines personnes parviendront à ramasser les bribes de leur vie, d'autres, sans que cela soit de leur faute, trouveront que leur vie est irrévocablement altérée. Cette tragédie reflète celle de chaque victime d'acte criminel dans notre société, mises à part les différences suivantes: nous ne connaissons pas la plupart des victimes; celles-ci ne reçoivent pas une vague de soutien et de réconfort; il leur est difficile d'avoir accès à une aide professionnelle qualifiée et elles sont livrées à elles-mêmes pour essayer de se rétablir. 
Bien que certaines victimes d'actes criminels arrivent à reprendre une vie normale, d'autres n'y arrivent pas. Dans cet article, l'auteure ne citera pas de données statistiques et ne fera aucune référence à des documents à l'appui de ses découvertes et de ses conclusions, pour le simple fait qu'il existe très peu d'études et d'informations statistiques dans ce domaine. Notre société s'est peu penchée sur le sort des victimes d'actes criminels. On sait qu'il existe une pléthore d'études et de statistiques sur les agresseurs dont on peut s'inspirer; on peut également citer un grand nombre de services et de programmes axés sur la réhabilitation des contrevenants. Par contre, très peu de données sur les victimes d'actes criminels sont disponibles, et les services et programmes visant à aider les victimes à se remettre de leur traumatisme sont extrêmement rares.

\section{LES RÉACTIONS DES VICTIMES D'ACTES CRIMINELS}

L'auteure, une travailleuse sociale spécialisée dans le traitement des victimes d'actes criminels et le syndrome du choc post-traumatique, a rencontré plusieurs centaines de victimes au cours des trois dernières années. Elle a noté le fait suivant: qu'il s'agisse de viols, de hold-up, d'agressions physiques, d'attaques à main armée, à arme blanche ou à arme à feu et même d'effraction, les victimes souffrent des conséquences du crime, non seulement pendant qu'il se déroule et immédiatement après, mais pendant plusieurs années. Même s'il n'y a pas de blessures physiques, il y a des blessures psychologiques. Les symptômes de ces blessures sont inhabituels et bouleversants. Il est terrifiant de perdre le contrôle de son corps et de sa vie et de s'apercevoir que l'on n'est pas capable de fonctionner normalement. Il est décourageant d'avoir devant soi la perspective d'un lent rétablissement. Il est également effrayant de ne pas comprendre pourquoi l'on n'est pas capable de se rétablir, malgré tous les efforts déployés pour être comme avant.

Pour beaucoup de victimes, ce qu'elles endurent après le crime est pire que l'acte criminel lui-même, même quand celui-ci a déjà été très pénible. Elles vivent un cauchemar permanent dont l'acte criminel a marqué le début.

Au moment de l'acte criminel, la victime n'a pas le temps de se préoccuper de ses émotions ou de se rendre compte de ce qui se passe. Cela se produit après. Les réactions pendant l'acte criminel sont d'ordre instinctif, comme dans toute autre situation imprévue où la vie est en danger. Toute l'attention de la victime est concentrée sur sa propre survie. Le corps est soumis à une tension aiguë et réagit en voulant à la fois lutter et fuir. La victime fonctionne par automatismes, comme si elle était devenue un robot. Les émotions sont momentanément inexistantes ${ }^{3}$. 
Choc physique, confusion et apathie sont les réactions dont souffre la victime. Elle est plongée dans un état de "peur paralysante». Comme dans toute autre situation de stress aigu, de l'adrénaline se répand à travers le corps, le corps a tendance à évacuer les matières qui le gênent, certains sens peuvent devenir très aiguisés alors que d'autres semblent "s'éteindre», les battements du cour s'accélèrent, puis la victime souffre d'hyperventilation, de transpiration et finalement d'épuisement ${ }^{4}$.

Deux victimes, en cours de traitement avec l'auteure, décrivent leur expérience. L'une d'elles, victime d'un hold-up, dit: «J'ai fait exactement ce qu'il $\mathrm{m}$ 'a dit de faire. J'étais paralysé. Je ne pouvais rien faire pour m'échapper. Je ne pouvais même pas penser. C'était comme si j'étais en train de regarder une pièce de théâtre, sauf que j'y participais». Une autre, victime d'une fusillade, dit: «Ça c'est passé si vite. Je l'ai vu entrer avec un revolver. Je savais qu'il allait tirer. Je n'ai pas eu le temps de penser. J'ai essayé de le désarmer. Je ne sentais rien. Il a tiré sur moi. C'était comme si tout ce qui se passait arrivait à quelqu'un d'autre, même si je savais que ça m'arrivait à moi».

Immédiatement après l'incident, le corps réagit physiquement au stress auquel il a été soumis. Trembler, pleurer, avoir des nausées, des vertiges, se sentir au bord de l'évanouissement, avoir les jambes tremblantes, des palpitations, des maux de tête et se sentir totalement épuisé, sont des réactions physiques fréquentes ${ }^{5}$.

En même temps, les émotions refont surface. Le choc, la peur, l'anxiété, la colère et le désarroi apparaissent. Au fur et à mesure que la victime réalise ce qui s'est passé, la réaction émotive devient de plus en plus aiguë.

Pendant les jours et les semaines qui suivent, les victimes souffrent de divers symptômes physiques et psychologiques en réaction au stress: maux de tête, sueurs froides, problèmes digestifs, fatigue, manque d'énergie, douleur musculaire, problèmes menstruels, changements dans les habitudes sexuelles, et troubles du sommeil. Les cauchemars et les récurrences (flashback) sont fréquents. Les victimes éprouvent de la difficulté à se concentrer et souffrent de pertes de mémoire. La panique, l'anxiété, la tristesse, la dépression et la culpabilité deviennent des sentiments courants. Elles présentent habituellement une indifférence émotive, c'est-à-dire qu'elles essaient de refouler les sentiments réels parce que la douleur est trop grande. Un sentiment d'impuissance se développe et la perte de contrôle sur leur corps et sur leurs émotions augmente ${ }^{6}$.

Chez bon nombre de personnes, l'état de détresse émotive aiguë et les malaises physiques commencent à s'estomper après quelques jours. Cependant, les réactions ne disparaissent pas complètement. Pour la majorité 
des victimes, les symptômes et les réactions, dont l'intensité varie, se font encore sentir durant des semaines, des mois et même des années après l'acte criminel.

\section{LES STADES DE RÉTABLISSEMENT}

Le rétablissement des victimes est souvent long et difficile. La réaction à une situation de crise comporte plusieurs étapes qui mènent au rétablissement. Bien qu'on ait donné à ces stades différents noms, on s'entend pour dire qu'il y a quatre étapes principales dans le processus: le choc, la rétrospective, la reprise en charge et le rétablissement. Pendant le stade de choc, les victimes sont en état de crise. Le déni est une réaction fréquente. Les victimes se sentent vulnérables, impuissantes, seules et dépossédées. Durant le stade de rétrospective, les victimes commencent à lutter pour s'adapter à la situation de violation et procèdent à la réunification de leur personnalité. Elles sont parfois capables de faire face à leur douleur. Parfois aussi, elles ont besoin de nier l'étendue des dommages de cette épreuve et des répercussions qu'elle a eues dans leur vie. Elles doivent faire face à des sentiments et des réactions qui les dépassent. Elles sentent souvent qu'elles perdent le contrôle. Le stade de reprise en charge est caractérisé par le fait que l'expérience douloureuse de l'acte criminel est désormais intégrée à leur vie au même titre qu'une autre expérience. Elles la considèrent sous un angle qui leur apparaît logique. Pendant le stade de rétablissement, les victimes ramassent les bribes de leur vie brisée et passent à autre chose?

Certaines victimes se remettent très bien de leur traumatisme. D'autres ont plus de mal à contrôler leurs symptômes. Certaines n'éprouvent que peu d'effets, d'autres, au contraire, souffrent de réactions très violentes. Chez certaines, il semble d'abord que le rétablissement soit en bonne voie, puis elles entrent soudain dans une phase de rémission et de rechute. D'autres ne subissent aucune rechute. Cependant, la majorité des victimes d'actes criminels souffrent de symptômes aigus pendant plusieurs mois.

Au cours des différents stades de rétablissement, les progrès sont souvent inégaux. Certaines personnes n'ont pas de chance et leur souffrance est longue et pénible. Le processus de rétablissement peut prendre des années, ce qui est souvent le cas par exemple en ce qui concerne les victimes d'agression sexuelle ou de vol à main armée. Certaines victimes s'en tirent très mal. Selon elles, leur vie est détruite. Elles ne peuvent se remettre et continuer à vivre normalement. Sans que ce soit de leur faute, elles souffrent de réactions aiguës ou chroniques durant les mois qui suivent l'acte criminel. Bon nombre d'entre 
elles souffrent du syndrome du choc post-traumatique, caractérisé par des troubles d'anxiété aigus et complètement débilitants ${ }^{8}$.

Les patients atteints de ce syndrome souffrent de souvenirs importuns qui se manifestent sous la forme de récurrences (flash-back), de cauchemars et de rêves éveillés. Ceux-ci se déroulent souvent au ralenti. La personne a l'impression d'avoir perdu le contrôle de son esprit ou de «devenir folle». Les victimes se sentent alors honteuses et angoissées. Elles éprouvent une peur terrible à l'idée que d'autres choses terrifiantes pourraient leur arriver. Elles ont honte de se «sentir faibles» et de ne pas pouvoir contrôler leurs émotions et leurs réactions.

Il se peut qu'elles souffrent d'hébétude psychique ou de déni. Elles ont l'air de bien aller, de fonctionner normalement. Elles essaient de se convaincre que tout va bien, et s'efforcent de remettre leur vie sur pied aussi vite que possible. Pour ce faire, les victimes refusent de reconnaître les répercussions accablantes du traumatisme. Les victimes du syndrome du choc posttraumatique souffrent aussi de stimulation du système autonome, ce qui les empêche de contrôler le niveau de leur tension psychique; elles se plaignent de symptômes tels que troubles du sommeil, hypervigilance, nervosité exacerbée face à des événements normaux, affaiblissement de la mémoire, difficultés à se concentrer, et cherchent à éviter les événements qui pourraient raviver les souvenirs du traumatisme".

Les symptômes du choc post-traumatique sont dévastateurs. Les victimes de cette réaction aiguë face à leur traumatisme sont souvent démunies psychologiquement. Elles sont incapables de fonctionner, même à un niveau élémentaire. Elles n'arrivent pas à faire les choses qui, avant, étaient simples. Sortir de chez elles et être confrontées aux autres sont désormais des tâches accablantes. Aller au travail est impossible. Les victimes du syndrome du choc post-traumatique n'éprouvent aucun plaisir à vivre.

Le rétablissement des victimes dépend de deux facteurs déterminants: le genre de traitement que les victimes reçoivent après l'acte criminel et la rapidité avec laquelle elles y ont accès. Elles peuvent se rétablir si elles bénéficient du traitement adéquat. La plupart d'entre elles peuvent se remettre des pires effets, apprendre à faire face à ce qui leur est arrivé et, après un certain temps, reprendre le cours normal de leur vie.

Cependant, il est certain que leur vie s'en trouve modifiée pour toujours. Ceux et celles qui ont surmonté leur traumatisme sont capables, après plusieurs années, de regarder en arrière et de considérer leur lutte comme une expérience positive d'épanouissement. Quoi qu'il en soit, ces victimes n'oublieront jamais. Elles n'oublieront jamais leur blessure et leur profonde souffrance. 
Elles n'oublieront jamais le nombre de problèmes qu'elles ont dû affronter. Elles n'oublieront jamais que tout cela a eu lieu sans qu'elles n'y soient pour rien. Elles n'oublieront jamais leur douleur. Une victime m'a dit un jour:

«C'est difficile pour moi de croire à quel point cette agression a détruit ma vie, même si maintenant je commence à me sentir mieux. Avant, j'étais une personne qui fonctionnait bien, $j$ 'étais indépendant et $j$ 'avais confiance en moi. Je me sentais bien dans ma peau. Ça allait bien dans ma vie. Je réglais facilement mes problèmes. J'étais si fort. Et pourtant, il (l'agresseur) m'a détruit. Un incident qui n'a même pas duré cinq minutes m’a transformé en une nullité, m'a rendu apeuré, dépressif et incapable de fonctionner normalement. Oui, je vois bien que ça va mieux maintenant. Quelque part, je sais que je vais m'en remettre. Mais pourtant, ça fait déjà presque quatre ans et j'ai encore tellement de choses à régler. Je pense que pour finir, c'est une expérience qui m'aura enrichi, mais je ne pourrai jamais oublier... ou bien lui pardonner pour les quatre années de ma vie qu'il m'a fait perdre, et combien il m'a fait souffrir.»

\section{LE SYNDROME DU CHOC POST-TRAUMATIQUE ET LES VICTIMES D'ACTES CRIMINELS}

En tant que société, nous n'avons pas fait beaucoup d'efforts pour reconnaître la souffrance des victimes d'actes criminels. Nous avons nié pendant trop longtemps les profondes répercussions de l'acte criminel dans la vie des victimes. En fait, nous nions l'existence de leur supplice et l'intensité de leur souffrance. Nous voulons qu'elles oublient. Nous voulons qu'elles se rétablissent et continuent à vivre. Nous les voulons de nouveau normales.

Pourtant, en tant que société, nous ne les aidons pas à se rétablir. Nous refusons de considérer leur souffrance comme une réaction normale à un traumatisme. Nous rendons souvent la situation encore plus difficile qu'elle ne l'est. Au lieu de leur offrir des services pour les aider à se rétablir, nous nous détournons d'elles et nous leur reprochons de ne pas être capables de s'en sortir.

Notre attitude vis-à-vis des victimes d'actes criminels est en fait la même que celle adoptée par les Américains face aux vétérans de la guerre du Viêtnam. De 1964 à 1973 dans le sud-est asiatique, environ un million de soldats furent témoins de combats actifs et furent confrontés à des situations dangereuses, où leur vie était en péril. Près de 500000 vétérans des combats subirent de graves réactions psychologiques, dues au traumatisme qu'ils avaient vécus. Ces réactions ont été identifiées comme le syndrome du choc post-traumatique (Post-traumatic stress syndrome, ou PTSD) ${ }^{10}$. 
Pendant de nombreuses années, la société américaine a nié et ignoré la souffrance des vétérans de la guerre du Viêt-nam. Il a d'abord fallu que des professionnels en santé mentale constatent des symptômes similaires chez des victimes de traumatismes autres que les combats, pour que le syndrome du choc post-traumatique soit finalement identifié. Au cours des années 70 , des accidents d'avion, des catastrophes naturelles, des incendies, des attentats terroristes contre des populations civiles et autres calamités se sont produits en grande quantité. On a constaté que chez les victimes de ces catastrophes, les réactions étaient similaires à celles remarquées chez les vétérans du Viêtnam. À la même époque, les traumatismes dus au viol, à la violence familiale, aux agressions sexuelles envers les enfants faisaient leur apparition dans les publications professionnelles. Les symptômes comportementaux de ces victimes étaient presque identiques.

C'est dans la publication de 1980 du Guide des diagnostics, le DMS-III (The Diagnostic and Statistical Manual of Mental Disorders)" que fut insérée une nouvelle rubrique: Les troubles de stress post-traumatique, aigus, chroniques, ou tardifs. Le syndrome du choc post-traumatique était finalement accepté comme étant le résultat réel et direct d'une confrontation à un traumatisme ${ }^{i 2}$. On avait longtemps attendu cette reconnaissance. Presque 40 ans plus tôt, en 1941, Kardiner l'avait décrite en la désignant de «physionévrose» ${ }^{13}$.

L'introduction de la notion du choc post-traumatique dans le DSM-III est importante. La réalité de la souffrance des victimes d'accidents, de désastres et de crimes de violence physique se voyait ainsi légitimée. Leur souffrance était acceptée médicalement et reconnue comme réelle.

Malgré cela, l'étendue de la souffrance des victimes est encore niée et sujette à des malentendus. Nombreux sont les médecins et les professionnels en santé mentale qui ne comprennent pas le syndrome du choc posttraumatique. Nombreuses sont les victimes exclues de la Loi d'indemnisation aux victimes d'actes criminels (au Québec, l'IVAC). Les services de consultation destinés aux victimes d'actes criminels sont difficiles à trouver. Alors que les victimes d'agression sexuelle, les femmes et les enfants victimes de violence conjugale, et les enfants victimes d'agression sexuelle et de violence physique se voient offrir un peu d'aide, sous forme de protection et de consultation, ce genre de service est virtuellement inexistant pour la personne attaquée dans la rue, pour le jeune poignardé dans le métro ou pour la victime d'un hold-up dans un dépanneur. 


\section{LES OBSTACLES AU RÉTABLISSEMENT}

La souffrance des victimes d'actes criminels est souvent aggravée par les personnes qui les entourent pendant les heures, les jours et les mois qui suivent l'agression. En effet, après des années de consultation auprès des victimes d'actes criminels, l'auteure a conclu que le rétablissement des victimes est directement lié à la sympathie, au support et à l'aide au'elles reçoivent dans les minutes, les semaines et les mois aui suivent le crime. Si la police se montre compatissante et aimable lors de l'enquête, les victimes se sentiront épaulées. Si le personnel médical et hospitalier se préoccupe de leurs besoins et se montre attentionné, aimable et compréhensif tout au long de la prise en charge, elles se sentiront soutenues. Si on les informe immédiatement sur les programmes d'indemnisation offerts par l'IVAC et la CSST, si on leur indique précisément comment obtenir cette indemnisation, et si on leur dit qu'elles peuvent en bénéficier dans un délai rapide, le stress supplémentaire auquel elles pourraient être soumises sera diminué.

Dans la mesure où l'on pourra prévenir les victimes et leur famille du type de réactions qu'elles risquent de subir, elles ne s'inquiéteront pas autant quand elles se produiront. Si les victimes peuvent bénéficier immédiatement d'une aide psychologique continue de la part d'un ou d'une spécialiste en santé mentale, qui saura comment les aider à se rétablir, leur condition s'améliorera. Si elles peuvent partager leur expérience avec d'autres victimes qui s'en sont sorties, elles pourront envisager l'avenir avec espoir. Si la famille de la victime comprend ses réactions et qu'on l'aide à faire face à ses propres sentiments vis-à-vis de l'acte criminel et de la victime, familles et victimes se soutiendront mutuellement. Il existe des facteurs qui influent sur les chances et les possibilités de rétablissement de la victime.

Lorsque la victime n'est pas entourée de sympathie, de soutien et d'aide, sa souffrance est d'autant plus grande. Le psychiatre Martin Symonds, de la police de la ville de New York, décrit la douleur et la blessure infligées aux victimes par leur entourage pendant les jours et les mois qui suivent, comme étant une deuxième blessure, qu'il a appelée «blessure secondaire» ${ }^{14}$. Au cours de son travail avec les victimes d'actes criminels, l'auteure a pu constater que la plupart d'entre elles souffraient de blessures secondaires multiples. En outre, elle a remarqué que la «blessure secondaire» est souvent plus nuisible, du point de vue psychologique, que le traumatisme lui-même. De plus, elle a aussi noté que la «blessure secondaire» rend l'apparition du syndrome du choc post-traumatique plus probable.

Il y a à cela de bonnes raisons. Après l'attentat criminel, les victimes sont pleines d'espoir et sont prêtes à croire qu'elles vont se rétablir. Si leur 
psychisme, déjà meurtri, se voit encore une fois maltraité, elles deviennent découragées, fatiguées, désespérées voire détruites. Plusieurs victimes ont témoigné de leurs blessures secondaires: «J'étais bouleversé et dérangé après l'agression. C'était la première fois que quelqu'un braquait un revolver sur moi. Le policier a fait une blague à ce sujet. Il pensait peut-être que ça aiderait, mais il était tellement insensible à tout ce que je pouvais sentir. J'ai ressenti que l'incident était banalisé. Et ça a eu pour effet que je me suis replié sur moi-même». Une autre victime dit: «On m'a emmenée à l'hôpital, on m'a laissée dans une salle de consultation et on $m$ 'a dit de me déshabiller. Je me sentais sale. Je me sentais seule. J'avais froid. Un médecin est entré et a insisté pour m'examiner. Je le trouvais antipathique et froid. Je me suis repliée sur moi-même et je l'ai laissé m'examiner.»

Une victime d'un hold-up raconte: «Quelques jours après le hold-up, je ne me sentais toujours pas très bien. J'avais peur de retourner travailler au magasin de vidéo. Mon patron m'a dit que je devais reprendre mon travail, sinon il me renverrait. J'y suis allé, mais j'avais des nausées et je tremblais. Je n'ai pas pu rester. Il était furieux contre moi». Parfois les réactions des familles viennent aggraver les blessures secondaires:

«D'abord, ma famille m'a beaucoup aidé. Ils étaient tellement révoltés par ce qui m'était arrivé. On en a parlé. Mais après un moment, quand j'essayais d'en parler, ils changeaient de sujet. Ils essayaient de me distraire. J'ai commencé à sentir qu'ils ne comprenaient pas. Alors que les mois passaient, ils toléraient de moins en moins ma dépression. Ils m'ont dit de m'en sortir. Mes sentiments vis-à-vis d'eux ont changé. Je ne savais pas pourquoi ils étaient capables de rire. Je ne pouvais pas. Je me sentais si seul.»

\section{COMMENTAIRES SUR LES SERVICES ET LA CONSULTATION}

Si l'on veut que les victimes aient une chance de se rétablir du traumatisme de l'acte criminel, il faut mettre à leur disposition des services de consultation. Il faut pouvoir offrir plusieurs types de consultation. Les victimes ont besoin d'être informées sur les indemnisations qu'elles peuvent obtenir. Elles ont besoin d'aide pour remplir les formulaires de demande d'indemnisation. Elles ont besoin de savoir ce qui va se passer à la cour, et d'y être accompagnées au bon moment. Elles ont besoin đ'aide pour faire face aux problèmes concrets qui découlent directement de l'acte criminel. Si la victime doit déménager à cause du crime par exemple, elle aura besoin d'aide pour négocier la résiliation du bail.

Le gouvernement du Québec s'est penché sur le sort des victimes d'actes criminels et a ouvert des centres régionaux d'aide aux victimes d'actes cri- 
minels (CAVAC). Ces centres remplissent le rôle de service de première ligne. Ils offrent des services importants : ils informent et aident les victimes à remplir les formulaires d'indemnisation, les accompagnent à la cour, leur apportent leur soutien après le crime et leur indiquent les soutiens professionnels disponibles. Les CAVAC représentent certainement un pas dans la bonne direction, mais ils ne sont pas assez nombreux. Les centres fonctionnent avec un directeur ou une directrice à plein temps et une équipe de bénévoles, ce qui limite l'aide qu'ils peuvent fournir.

Les victimes d'actes criminels endurent une réelle tragédie, qui détruit leur vie. Elles sont souvent incapables de reprendre leur travail ou voient leurs performances professionnelles chuter. Pour beaucoup d'entre elles, les relations familiales se détériorent. Elles sont sujettes de plus en plus souvent à des problèmes de santé. Elles cessent leurs activités extérieures et se coupent de toute relation sociale. Elles sont affectées par des peurs paralysantes qui se transforment en réactions phobiques généralisées. Elles sont sujettes à des attaques de panique. Elles souffrent d'un sentiment de futilité et veulent mourir.

Notre société ne reconnaît pas l'intensité et la gravité de leurs blessures. Nous refusons de voir les dégâts malgré leur évidence. Au lieu d'apporter aux victimes les types d'interventions les plus spécialisées, les plus intensives et les plus professionnelles, nous faisons le contraire. Nous ne mettons à la disposition des victimes que très peu de services et parfois même aucun. Nous refusons de reconnaître la destruction psychologique opérée par le crime. Mais le pire est qu'au lieu de les soutenir, nous leur faisons des reproches. Nous leur reprochons de s'être trouvées au mauvais endroit, au mauvais moment. Nous leur reprochons la violence de leurs réactions. Nous guettons leurs failles ou leurs faiblesses psychologiques pour pouvoir expliquer leur incapacité de se débrouiller. Nous leur reprochons de ne pas se rétablir assez vite. Nous leur reprochons d'avoir changé pour toujours et de ne plus être comme avant. Nous leur reprochons les changements opérés dans les relations familiales et dans les amitiés, la modification de leur regard sur le monde. Nous souffrons du syndrome «blâmons la victime», qui n'entraînera que des dégâts supplémentaires.

Il nous faut modifier notre attitude : ne plus blâmer, mais accepter. Nous devons aussi pouvoir offrir d'autres sortes de services de consultation.

L'intervention en situation traumatisante est un de ceux-là. Cette intervention, mise en pratique après une expérience traumatisante significative, est destinée à réduire les symptômes liés au stress pendant et après le traumatisme. Le but de l'intervention en situation traumatisante est de réduire et de maîtriser les répercussions du traumatisme. Cela permet à l'individu 
de progresser plus rapidement à l'intérieur du processus normal de rétablissement lorsqu'il réagit normalement à des circonstances anormales. Cela lui permet de donner libre cours à ses émotions et analyse les répercussions personnelles du traumatisme. Quand ce traitement est pratiqué en groupe, il favorise l'établissement d'un soutien du groupe pour répondre à des conditions de traumatisme; les participants peuvent exprimer leur douleur; le sentiment illusoire de leur unicité s'en trouve réduit (je suis le-la seul-e à me sentir comme ça) ainsi que celui de leur anormalité (il doit y avoir quelque chose qui ne tourne pas rond en moi si je me sens comme ça). Cela sert aussi à protéger les personnes à risques, et met à leur disposition un ensemble de référence s'ils ont besoin d'autres services de consultation ${ }^{15}$.

La consultation individuelle, dispensée par des professionnel-le-s en santé mentale, devrait être mise à la disposition de chaque victime qui souffre de troubles légers ou sérieux, immédiatement après l'acte criminel, mais aussi durant les mois ou les années qui suivent. Quand cette intervention est prodiguée immédiatement après l'acte criminel, l'approche utilisée est l'intervention auprès de personnes en situation de crise. C'est une approche clinique orientée vers la réalité qui se concentre sur la crise, normalise les réactions et aide la victime à regagner son équilibre.

Afin de réduire l'étendue du traumatisme, la victime devrait bénéficier d'une intervention professionnelle aussitôt que possible après le traumatisme. L'auteure s'est aperçue que plus le traitement professionnel se faisait attendre, plus la période de rétablissement de la victime était longue et difficile.

Beaucoup de victimes ont besoin d'une psychothérapie de courte durée ou de longue durée. Cela dépend de l'intensité de leurs réactions et de la rapidité avec laquelle elles ont eu accès à une aide professionnelle. La thérapie doit être axée sur la réalité, elle doit soutenir le patient ou la patiente et être intensive. Elle doit être axée sur le traumatisme, la rupture qu'il a causée et doit conduire la victime au rétablissement.

Beaucoup de victimes ont besoin d'un traitement pendant les mois et les années qui suivent l'incident traumatique. De nombreux facteurs rendent pressant le besoin d'être traité. Souvent les victimes essaient de faire face toutes seules aux phases aiguës de la névrose post-traumatique, jusqu'à ce qu'elles deviennent profondément retirées en elles-mêmes ou même suicidaires. D'autres constatent que leurs symptômes ont disparu mais ils réapparaissent plus tard sous une forme encore plus grave. Pour beaucoup, il arrive qu'une situation de stress non liée au traumatisme, juste après le crime ou des années plus tard, provoque une réaction intense. Quels que soient leurs efforts, elles sont incapables de fonctionner comme avant. 
Même quand les victimes vont chercher de l'aide, leur condition ne s'améliore pas forcément. Les victimes se sentent souvent plus mal quand elles sont en traitement. Chez beaucoup de victimes en traitement auprès de psychanalystes réputés, de travailleurs sociaux ou de psychologues, on ne constate aucune amélioration. Cela n'est pas étonnant si les traitements ne se concentrent pas sur le fait que ces personnes sont des victimes d'actes criminels mais sur d'autres choses, telles que leur dépression ou leur anxiété. Il arrive souvent que le professionnel, ne comprenant pas le syndrome du choc post-traumatique, ne normalise pas ce qui se passe. De plus, ignorant le processus de rétablissement, il axe le traitement sur de faux problèmes. Les victimes ne sont souvent pas satisfaites de leur thérapeute, mais continuent le traitement parce qu'elles ne connaissent pas d'autres lieux ou ignorent quoi faire. Une victime dans cette situation a dit: «Le médecin insistait pour que je prenne des médicaments. Je me sentais abrutie. J'étais dans un groupe de thérapie avec des gens qui étaient déprimés et personne d'autre n'était victime d'acte criminel. Je me sentais malade. Quand j'ai essayé de parler de mes peurs, on m'a dit que la meilleure chose que je pouvais faire, c'était d'oublier le hold-up.» Quand cette femme est venue me voir, huit mois après le crime, et après avoir consulté un psychologue et un psychiatre pendant six mois, elle a commencé à pleurer. Personne ne lui avait dit que les symptômes et les réactions qu'elle éprouvait étaient typiques des victimes d'actes criminels. Personne ne lui avait donné l'occasion de parler de ce qui lui était arrivé. Les professionnels traitants avaient banalisé l'incident et provoqué une «blessure secondaire».

Plus l'intervention auprès des victimes qui souffrent des symptômes du choc post-traumatique sera rapide, plus elle sera efficace. Ceux et celles qui souffrent de névrose post-traumatique tardive vivent un rétablissement difficile, tout comme ceux et celles qui subissent des «blessures secondaires». Les victimes peuvent se rétablir et se rétablissent si on leur donne accès à un bon traitement. Quoi qu'il en soit, nous ne devons pas envisager le rétablissement en termes de jours ou de semaines mais en termes de mois et d'années. Cela conditionne l'engagement des professionnels traitants en santé mentale. Travailler avec les victimes demande beaucoup de patience, de compréhension et de compétence. Il faut faire face à de nombreuses questions au cours du traitement. À quel point devrait-on encourager la victime à reprendre son travail? Sachant que les rechutes qui frappent tant de victimes sont inévitables, comment allez-vous les aborder? Comment pouvez-vous continuellement encourager la victime quand les progrès sont si lents? Que faire face aux réactions de la famille, des amis, des médecins et des représentants de la Commission de la santé et de la sécurité au travail (CSST), qui s'attendent à des progrès rapides et font subtilement pression sur la victime, en lui donnant 
l'impression que la lenteur à laquelle elle se rétablit n'est pas normale? Comment convaincre les victimes qu'elles ne sont pas les seules, que ce qu'elles sont en train de vivre est affreusement douloureux, mais que c'est la même chose pour chaque victime d'acte criminel?

\section{LES GROUPES DE SOUTIEN ET D'ENTRAIDE POUR LES VICTIMES}

Bon nombre de victimes se posent les mêmes questions et s'attachent à résoudre les mêmes problèmes. De ce fait, le soutien de personnes qui ont vécu des expériences similaires devient aussi important que la consultation individuelle.

Il existe aux États-Unis plusieurs groupes de soutien et d'entraide. ll y en a très peu au Canada. Parmi les exemples de tels groupes, on peut citer MADD, MOTHERS AGAINST DRUNK DRIVERS (États-Unis) et VICTIMS OF VIOLENCE NATIONAL INC. (Ontario). MADD apporte son soutien aux parents dont les enfants ont été blessés ou tués par des conducteurs en état d'ébriété. C'est également un groupe de pression qui agit auprès du gouvernement pour que celui-ci sanctionne plus sévèrement les infractions de conduite en état d'ébriété. VICTIMS OF VIOLENCE NATIONALINC. est, quant à lui, un groupe de soutien et d'entraide ontarien qui rassemble les parents dont les enfants ont été assassinés et leur apporte soutien et réconfort.

L'auteure a été préoccupée par l'absence de tels groupes au Québec. Ses clients-es lui demandaient souvent: «Est-ce que d'autres victimes vivent la même chose que moi?... Est-ce que c'est normal?... Est-ce que je suis malade? Pourquoi est-ce que je ne vais pas mieux?... Je me sens si seul-e... personne ne sait comment je me sens..." Ce dont l'auteure se rendait bien compte, c'est du besoin ressenti par ces personnes de se voir confirmer, par d'autres qui auraient vécu des expériences semblables, que ce qu'elles ressentaient était normal. C'est pourquoi elle a fondé en février 1989, avec deux autres bénévoles, un groupe d'entraide et de soutien pour les victimes d'actes criminels appelé Victimes de crime. Le groupe a été formé dans le but d'aider les victimes à mettre en place un réseau de soutien, de briser leur isolement, de leur permettre de se rendre compte que ce qu'elles vivent est normal et qu'elles ne sont pas seules. L'auteure a tout d'abord pensé que ce groupe serait destiné à aider ses propres clients et clientes, mais il s'est avéré que de nombreuses victimes qui n'étaient pas en traitement pouvaient en bénéficier. Il avait été prévu que Victimes de crime serait un groupe d'entraide, mais le besoin d'un leadership professionnel s'est rapidement fait sentir. Les personnes qui participaient au groupe étaient souvent trop fragiles pour pouvoir communiquer avec les autres. Elles voulaient que la ou le thérapeute inter- 
vienne et contrôle. Le concept du groupe s'en est trouvé modifié: Victimes de crime est maintenant un groupe de support et prend souvent l'allure d'une thérapie de groupe.

Au début de 1990, le groupe a officiellement modifié son nom pour s'appeler Association victimes de crime de Montréal. À la suite de nombreuses requêtes de la part de la famille ou des amis de victimes, l'Association victimes de crime de Montréal a élargi ses services de façon à desservir également la famille et les amis de victimes, et a formé un groupe pour les membres de la famille et les amis des victimes d'homicide.

L'Association victimes de crime de Montréal se distingue du modèle des autres groupes de support de plusieurs façons. Elle accueille des victimes et des membres de la famille des victimes de toutes sortes de crimes: agression sexuelle, fusillade, prise d'otage, attaque à main armée, effraction, vol de sac à main ou voies de fait. Ces hommes et femmes de tous âges se rencontrent régulièrement dans le but d'être soutenus, compris, réconfortés et aidés dans leur processus de rétablissement.

L'expérience de l'Association victimes de crime de Montréal a prouvé à l'auteure l'importance d'un groupe de soutien pour certaines victimes d'actes criminels. Ce n'est pas pour tous ou toutes, et certaines victimes ne seraient pas en mesure d'y participer: leur capacité de faire partie d'un tel groupe et d'en tirer profit dépend pour beaucoup de l'étape où elles en sont rendues dans leur rétablissement. L'atmosphère des réunions est souvent chargée d'émotions, et c'est parfois trop pour certaines personnes. Pour d'autres, par contre, l'effet du groupe est quasi «magique». Un participant disait à la fin de sa première réunion: «Cela fait du bien de savoir que d'autres vivent la même chose que moi. Mais cela me fait peur, mon traumatisme date de deux mois, pour certains d'entre vous cela fait deux ans que c'est arrivé. Cela me fait peur de voir que ça peut prendre autant de temps avant que je sois rétabli».

\section{REJOINDRE LES VICTIMES D'ACTES CRIMINELS}

Les victimes d'actes criminels ne constituent pas un groupe facile à traiter ni même à atteindre. Avant le crime, beaucoup d'entre elles n'ont jamais eu besoin d'aide. Elles étaient indépendantes, autonomes et vivaient sans problèmes. Elles résistent donc souvent au traitement. Elles se croient capables de résoudre elles-mêmes leurs difficultés et de se rétablir par elles-mêmes. Elles sont très fières et éprouvent de la difficulté à chercher de l'aide. Elles sont très sensibles aux commentaires et aux sous-entendus qui peuvent venir des professionnels traitants. Elles ne font confiance au processus thérapeutique et ne s'y engagent qu'après une longue période. Elles ont l'impression d'être 
bizarres, comme si elles étaient excentriques, et ont honte de ce qu'elles perçoivent comme étant de la faiblesse. Elles ont beaucoup de mal à comprendre et à accepter ce qu'elles vivent, tout comme il leur est difficile de se voir comme elles sont maintenant plutôt que comme elles étaient avant le crime. Pourtant, le professionnel qui est compétent en victimologie et qui est à l'écoute de leurs problèmes peut les aider. Le professionnel devient alors plus que celui qui aide. Il devient souvent celui qui a sauvé leur vie, un ami.

Le travail parmi les victimes peut être satisfaisant; il peut aussi être frustrant. Nous commençons juste à comprendre ce qu'est le choc posttraumatique. Nous savons encore bien peu de choses sur les techniques et les interventions qui peuvent vraiment aider les victimes d'actes criminels. Il arrive souvent que, croyant les aider, nous fassions des choses qui leur font mal et augmentent leur souffrance. Il arrive que, sans nous en rendre compte, nous leur nuisions en leur faisant savoir, de manière plus ou moins subtile, que nous ne les comprenons pas, et en leur proposant des solutions simples pour un processus de rétablissement très complexe.

Les victimes d'actes criminels ont besoin du traitement le plus éprouvé, le plus complet et le plus élaboré qui soit. Elles ont besoin d'être traitées par des professionnels qui prennent le temps et ont la patience de travailler avec elles durant de longues heures, qui expérimentent des approches nouvelles de traitement. Elles ont besoin de l'aide des professionnels qui comprennent la névrose post-traumatique dans son ensemble, ainsi que les différents stades du processus de rétablissement. Elles ont besoin de divers types de traitement: travail en situation de crise, services d'information, psychothérapie destinée aux victimes et groupe de soutien. Elles ont besoin que des gens concernés et compétents leur offrent de l'aide, et leur en donnent sans compter.

Malheureusement, les choses ne se passent pas ainsi. Elles doivent souvent attendre des mois avant d'être traitées. Les diagnostics sont fréquemment faux. La plupart du temps, on leur donne des médicaments qui ne leur conviennent pas et qui aggravent leurs problèmes. On leur fait souvent subir un traitement inapproprié et leur condition empire au lieu de s'améliorer. Pour finir, au lieu de prendre au sérieux les victimes d'actes criminels, nous banalisons leurs problèmes, nions l'étendue de leur souffrance et refusons d'admettre l'effet dévastateur du crime sur leur vie.

\section{CONCLUSION}

Jusqu'à ce que notre société ait acquis une meilleure compréhension des problèmes des victimes d'actes criminels, nous continuerons à gêner leur rétablissement et ainsi à leur nuire. Il semble qu'il y ait un malentendu quant 
aux moyens nécessaires pour aider les victimes. Nous pensons qu'une équipe de volontaires ayant reçu une formation minimale suffit à répondre aux besoins des victimes. Les volontaires et les CAVAC qui sont actuellement mis en place au Québec sont essentiels. Pourtant les CAVAC ne devraient représenter, dans l'ensemble des services offerts aux victimes, qu'un maillon de la chaîne. Pour traiter les victimes qui sont réellement affectées par le crime sur le plan psychologique, on doit constituer des équipes multidisciplinaires, spécialement formées et intéressées à traiter toutes les sortes de victimes d'actes criminels. On doit former des professionnels. On doit entreprendre des recherches sur les différentes interventions de traitements. On doit fonder des groupes de soutien.

Qu'il s'agisse d'un hold-up, d'une attaque à main armée dans la rue, d'une attaque à arme blanche ou à arme à feu, d'une agression sexuelle, d'une attaque à main nue, ou d'une tentative de meurtre, chaque victime souffre de réactions qui ravagent sa vie. La famille d'une victime de meurtre. les parents dont les enfants ont été agressés sexuellement par un tiers, les rescapés d'un accident causé par un conducteur en êtat d'ébriété ou les familles de ceux qui ont été tués par un conducteur en état d'ébriété, enfïn, les témoins d'un crime violent, tous sont victimes. Tous ces gens ont besoin de recevoir l'aide de professionnels en santé mentale, sympathiques, compatissants et patients, compétents et formés dans les domaines de la victimologie, du choc post-traumatique, de la pharmacologie et de la psychothérapie.

\section{RÉFÉRENCES}

1. SOURCE: ministère de la Sécurité publique.

2. STATISTIQUES DE LA CRIMINALITÉ (1988, 1989), Service de police de la CUM.

3. SELYE, Hana (1974), Stress sans détresse, Montréal, La Presse.

4. et 5. NATIONAL ORGANIZATION FOR VICTIM ASSISTANCE (février 1987) The Crisis Reaction. Washington, DC.

6. L'ASSOCIATION QUÉBÉCOISE PLAIDOYER-VICTIMES INC. (février 1987), Guide d'intervention auprès des victimes d'actes criminels, Montréal.

7. BARD, MORTON \& SANGREY, DAWN (1986), The Crime Victim's Book, New Jersey. Citadel Press, p. 32-52.

8. Ibidem, p. 47. Note: l'auteure regrette de n'avoir pu trouver aucune documentation sur la proportion de victimes qui sont atteintes de ce syndrome. ni sur les taux de guérison.

9. FRIEDMAN, R., FRAMER M. \& SHEARER, D. (sept/oct 1988), «Early Response to Post-traumatic Stress" in EAP Digest, p. 45-56. 
10. WILliAMS, T. (1987), Post-traumatic Stress Disorders : A Handbook for Clinicians, Ohio, Disabled American Veterans.

11. AMERICAN PSYCHIATRIC ASSOCIATION, Diagnostic and Statistical Manual of Mental Disorders, third edition and third edition-revised. 1980. Bien que le diagnostic soit dans le DMS-III les troubles de stress post-traunatique. le terme névrose post-traumatique est aussi utilisé. L'auteure préfere le terme «névrose", qui représente une réaction à celui de troubles qui équivaut à une maladie.

12. WILliAMS, T. (1987), Post-traumatic Stress Disorders: A Handbook for Clinicians, Ohio, Disabled American Veterans.

13. KARDINER, A. (1941), The traumatic Neuroses of War, New York, Hoeber.

14. FRIEDMAN, R. FRAMER, M. \& SHEARER. D. (sept/oct 1988), «Early Response to Post-traumatic Stress" in EAP Digest.

15. CHATHAM POLICE FORCE (1988), C.I.S. DEBRIEF, (vidéo), Chatham, Ontario. 